\title{
Daphnetin suppresses experimental abdominal aortic aneurysms in mice via inhibition of aortic mural inflammation
}

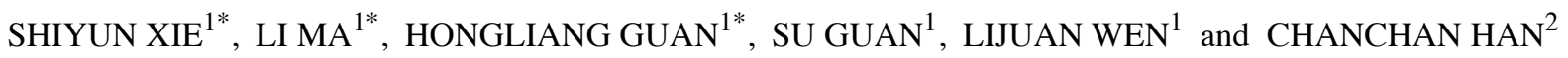 \\ ${ }^{1}$ Department of Vascular Surgery, Shandong Shanxian Central Hospital, Shanxian, Shandong 274300; \\ ${ }^{2}$ Department of Ultrasound, Tengzhou Central People's Hospital, Tengzhou, Shandong 277500, P.R. China
}

Received February 12, 2020; Accepted July 21, 2020

DOI: $10.3892 / \mathrm{etm} .2020 .9351$

\begin{abstract}
Rupture of abdominal aortic aneurysm (AAA) is a devastating event that can be prevented by inhibiting the growth of small aneurysms. Therapeutic strategies targeting certain events that promote the development of AAA must be developed, in order to alter the course of AAA. Chronic inflammation of the aortic mural is a major characteristic of AAA and is related to AAA formation, development and rupture. Daphnetin (DAP) is a coumarin derivative with anti-inflammatory properties that is extracted from Daphne odora var. However, the effect of DAP on AAA development remains unclear. The present study investigated the effect of DAP on the formation and development of experimental AAAs and its potential underlying mechanisms. A mice AAA model was established by intra-aortic infusion of porcine pancreatic elastase (PPE), and mice were intraperitoneally injected with DAP immediately after PPE infusion. The maximum diameter of the abdominal aorta was measured by ultrasound system, and aortic mural changes were investigated by Elastica van Gieson (EVG) staining and immunohistochemical staining. The results demonstrated that DAP significantly suppressed PPE-induced AAA formation and attenuated the depletion of aortic medial elastin and smooth muscle cells in the media of the aorta. Furthermore, the density of mural macrophages, $T$ cells and B cells were significantly attenuated in DAP-treated AAA mice. In addition, treatment with DAP resulted in a significant reduction in mural neovessels. These findings indicated that
\end{abstract}

Correspondence to: Dr Chanchan Han, Department of Ultrasound, Tengzhou Central People's Hospital, 181 Xingtan Road, Tengzhou, Shandong 277500, P.R. China

E-mail: 3444658726@qq.com

Dr Lijuan Wen, Department of Vascular Surgery, Shandong Shanxian Central Hospital, 1 Wenhua Road, Shanxian, Shandong 274300, P.R. China

E-mail: 596873378@qq.com

*Contributed equally

Key words: abdominal aortic aneurysm, daphnetin, inflammation, mural angiogenesis, treatment
DAP may limit the formation and progression of experimental aneurysms by inhibiting mural inflammation and angiogenesis. These data confirmed the translational potential of DAP inclinical AAA inhibition strategies.

\section{Introduction}

Abdominal aortic aneurysm (AAA) is a lethal, age- and sex-related chronic inflammatory degenerative disease, with a prevalence ranging from $5-10 \%$ amongst men $>65$ years old, and increasing with age (1-3). Recently, endovascular aneurysm repair (EVAR) is a new technology that is used to treat patients with AAA when the anatomy is suitable $(4,5)$. Greenhalgh et al (4) reported that the 30-day mortality of AAA in the EVAR group was 1.7 vs. $4.7 \%$ in the open repair group within British hospitals. Although treatment methods for AAA have improved significantly in the last few decades, the mortality rate of patients with ruptured AAA remains very high worldwide $(5,6)$. At present, surgical repair is an effective treatment for advanced AAAs (diameter $>55 \mathrm{~mm}$ ). Public screening programmes frequently detect AAA at an early stage (7). However, surgical repair does not provide a significant benefit for AAA at an early stage (8), and the current practice is to watch and wait until the diameter exceeds $55 \mathrm{~mm}$, resulting in a risk of rupture or subsequent complications. AAA has a natural process of progressive growth, which indicates that early stage AAA can grow and subsequently require surgical treatment (9). To date, no pharmacological inhibition strategies have been effective in suppressing AAA development or eventual rupture (7). Discovering novel drugs that could inhibit the development of AAA is therefore crucial.

AAA is characterized by increased mural inflammation, neoangiogenesis, degeneration of smooth muscle cells (SMCs), degradation of extracellular matrix, activation of matrix metalloproteinases (MMPs) and accumulation of intraluminal thrombi (10). Therapeutic strategies for stopping or decelerating AAA progression must target the underlying events that promote its development (11). Mural inflammation has been implicated in AAA formation, development and rupture. Lindberg et al (12) reported that numerous inflammatory biomarkers are significantly elevated and positively correlated with aortic diameter in abdominal aortic aneurysms. Furthermore, anti-inflammation-based therapy has been demonstrated to attenuate aneurysmal development in 
AAA models (11,13-15). Xiong et al (13) demonstrated that blocking TNF- $\alpha$ expression can attenuate aneurysm formation in a murine model. Li et al (11) reported that cold-inducible RNA-binding protein (CIRP) is a novel proinflammatory cytokine, and that anti-CIRP-based therapy could attenuate aneurysm formation by inhibiting mural inflammation in an experimental murine model. Numerous studies have therefore focused on exploring anti-inflammatory agents that could alleviate AAA development. Previous studies have demonstrated that some natural products can be used to treat coronary heart disease, atherosclerosis, diabetes mellitus due to their powerful anti-inflammatory activities (16-18). Furthermore, previous studies reported that natural products have antioxidant and anti-inflammatory effects. Fan et al (19) found that curcuma, which is the source of the spice turmeric widely used in Asian countries, can attenuate rat thoracic aortic aneurysm formation. In addition, Hao et al (16) reported that curcumin attenuates AAA by inhibiting the inflammatory response in a murine model. Importantly, Kaluza et al (20) demonstrated that an anti-inflammatory diet is associated with a reduced risk of AAA, which is an association that was even more significant for AAA rupture. Natural products are therefore considered as ideal sources of potential agents capable of managing AAA because of their low toxicity and the absence of clear side effects.

Daphnetin (7,8-dihydroxycoumarin; DAP) is a natural anti-inflammatory, anti-oxidative and anti tumour product (21-25) that is extracted from Daphne odora var. Liu et al (26) reported that DAP has some protective effects on severe acute pancreatitis in a rat model by inhibiting the expression of inflammatory cytokines. Subsequently, DAP was found to prevent and treat numerous diseases, including cerebral ischaemia/reperfusion injury, rheumatoid arthritis, colitis and endotoxin-induced lung injury, due to its powerful anti-inflammatory activities (24,27-32). At present, DAP is an anti-inflammatory agent used in some inflammation-related diseases (33-35). Li et al (33) reported that DAP inhibited inflammation in the systemic lupus erythematosus murine model via inhibition of $\mathrm{NF}-\kappa \mathrm{B}$ activity. Wang et al (34) demonstrated that DAP alleviated experimental autoimmune encephalomyelitis via regulating dendritic cell activity. Chronic inflammation of the aortic mural is a major characteristic of AAA and is related to AAA formation, development and rupture (1). However, the anti-inflammatory effect of DAP on AAA remains to be determined.

The present study hypothesized that DAP could prevent aneurysm development by inhibiting the inflammatory response. To investigate this hypothesis, this study established an AAA mice model induced by intra-aortic infusion of porcine pancreatic elastase (PPE) and the effect of DAP on AAA mice were evaluated.

\section{Materials and methods}

Experimental animals. The present study used 14 male C57BL/6 mice weighing 24-27 g (Beijing Vital River Laboratory Animal Technology Co., Ltd.) aged 8-10 weeks. All experimental protocols were approved by the Medical Ethics Committee of Shandong Shanxian Central Hospital. All animals were cared for in accordance with the recommendations of the Guide for the Care and Use of Laboratory Animals of the National Institutes of Health (36).

Animal model establishment. The AAA model was induced by intra-aortic infusion of type I porcine pancreatic elastase (PPE) as described previously (3,37-39). In the present study, mice were anesthetized using $4 \%$ isoflurane prior to surgery followed by $2.5 \%$ isoflurane to maintain the anaesthesia. Once mice were anesthetized, a vertical midline abdominal incision was performed. After exposing the posterior abdominal wall, the abdominal aorta from the left renal vein to the bifurcation was isolated, and the lumbar arteries were ligated under sterile conditions. The proximal and distal abdominal aortas were temporarily blocked with 6-0 silk sutures. Subsequently, a polyethylene catheter (PE-10, Instech Laboratories, Inc.) was inserted into the controlled aorta above the bifurcation, and the aorta was infused with type $1 \mathrm{PPE}(1.5 \mathrm{U} / \mathrm{ml}$ in saline; Sigma-Aldrich; Merck KGaA) for 5 min under constant pressure $(100 \mathrm{~mm} \mathrm{Hg})$.

During the 14 days following the operation, mice were intraperitoneally injected with DAP or an equal volume of vehicle every day. On the 14th day after the operation, mice were euthanized under $\mathrm{CO}_{2}$ exposure (flowrate of $\mathrm{CO}_{2}, 2 \mathrm{l} / \mathrm{min}$; air displacement rate, $20 \% / \mathrm{min}$ ). Death was confirmed by the absence of breathing, pulse, corneal reflex, response to firm toe pinch, heart beat and respiratory sounds, the graying of the mucous membranes and rigor mortis. Exception of rigor mortis, none of these signs can independently confirm death. Blood was collected after mice euthanasia cardiac puncture. Blood was collected $(0.6 \mathrm{ml})$ and stored in $1.5 \mathrm{ml}$ tube for $2 \mathrm{~h}$ at $4^{\circ} \mathrm{C}$. Then, $0.2 \mathrm{ml}$ serum was collected after centrifugation at $3,000 \mathrm{x} \mathrm{g}$ at $4^{\circ} \mathrm{C}$ for $10 \mathrm{~min}$. Aortic infused segments (about $10 \mathrm{~mm}$ long) were also collected, embedded in optimal cutting compound media (cat. no. 4583; Sakura Finetek USA, Inc.) and then stored in $-80^{\circ} \mathrm{C}$.

Experimental groups and DAP treatment. Mice were randomly assigned into two groups as follows: The AAA+ vehicle group $(n=7$, surgery was not performed on one mouse as a abdominal aorta aberrance which was identified via ultrasound, which measured the maximum diameter of the abdominal aorta before surgery) and the AAA+DAP group $(n=7)$. Briefly, DAP (purity $>98 \%$ ) was obtained from Sigma-Aldrich; Merck KGaA. DAP was dissolved at the concentration of $1 \mathrm{mg} / \mathrm{ml}$ in a solvent composed of $5 \%$ DMSO and $95 \%$ saline. Mice were intraperitoneally injected with DAP (20 mg/kg/day) or an equal volume of vehicle immediately after PPE infusion and lasted for 14 days. The dose of DAP was determined according to a previous study demonstrating that improved survival in a rodent cerebral ischaemia/reperfusion model (24). Aortic segments were subsequently collected on day 14 following surgery operation.

Aortic size measurements via ultrasound. The maximum diameter of the abdominal aorta was measured in anesthetized mice by using a $30 \mathrm{MHz}$ ultrasound system (VisualSonics, Inc.) before surgery and on days 3, 7 and 14 following surgery. Two experienced operators who were blinded to study group assignments independently completed the full-scale measurement and quantitative analysis of the ultrasonic data. AAA 
was defined as a $50 \%$ or greater increase in infrarenal aortic diameter compared with the baseline assessment (Vevo 770 ultrasound system).

Elasticavan Gieson staining. Aortas were collected on the 14th day after the operation and embedded in a straight strip shape in optimal cutting compound media (cat. no. 4583; Sakura Finetek USA Inc.). To observe changes in the aortic tissues at the morphological level, $8-\mu \mathrm{m}$ sections of aortic tissue were sectioned and fixed with cold acetone for $8 \mathrm{~min}$ at $4^{\circ} \mathrm{C}$. To evaluate the integrity of elastin, aortic tissue sections were stained with Elasticavan Gieson (EVG; cat. no. G1042, Wuhan Servicebio Technology Co. Ltd.) according to the manufacturers' protocol. As previously described, the analysis of medial elastin destruction was graded from mild (I) to severe (IV) (3).

Immunohistochemical staining. Immunohistochemical staining was performed as described previously (3). Briefly, 8- $\mu \mathrm{m}$ sections of aortic tissue were prepared as afore mentioned for EVG staining. The sections were incubated with antibodies against $\alpha$-smooth muscle actin ( $\alpha$-SMA; $1: 400$; cat. no. ab32575; Abcam), CD68 (1:400; cat. no. ab125212, Abcam), CD8 (1:100; cat. no. ab22378; Abcam), B220 (1:100; cat. no. ab64110; Abcam) and CD31 (1:200; cat. no. ab28364; Abcam) overnight at $4^{\circ} \mathrm{C}$. Then, sections were incubated with a biotinylated secondary antibody kit (cat. nos. PV-9001 and PV-9004; Beijing zhongshan Jinqiao Biotechnology Co., Ltd.), and immune complexes were visualized using a 3-amino-9-ethylcarbazole (AEC) peroxidase substrate kit (cat. no. A2010; Beijing Solarbio Science \& Technology Co., Ltd) according to the standard procedure. Subsequently, the sections were counterstained with haematoxylin. After dehydration overnight at $25^{\circ} \mathrm{C}$ incubator, clearing and mounting using aqueous non-fluorescing mounting media (cat. no. HS-106; National Diagnostics), sections were visualized under x10 magnification by microscopy (CX31; Olympus Corporation). The degeneration of SMCs was graded from mild (I) to severe (IV) as described previously (3). The infiltration of macrophages was graded as follows: i) I, infiltration limited to the adventitia or $<1 / 4$ of the aortic circumference; ii) II, infiltration involving the medial layer and $\leq 1 / 2$ of the aortic circumference; iii) III, infiltration involving the medial layer and $>1 / 2$ and $\leq 3 / 4$ of the aortic circumference; and iv) IV, infiltration involving all aortic layers and expanded $>3 / 4$ of the aortic circumference. Mural T cell infiltration, B cell infiltration and angiogenesis were quantified as $\mathrm{CD}^{+}, \mathrm{B} 220^{+}$and $\mathrm{CD} 31^{+}$ blood vessels, respectively, per aortic cross section analysis.

Statistical analysis. Data are presented as the means \pm standard deviation. Statistical analysis was performed using GraphPad Prism 6.0 (GraphPad Software, Inc.). Student'o t-test was used to evaluate the significance of differences between two groups. $\mathrm{P}<0.05$ was considered to indicate a statistically significant difference.

\section{Results}

DAP treatment attenuates experimental AAA formation and development. The maximum aortic diameter was measured with by ultrasound before the operation and on days 3, 7 and 14 after operation (Fig. 1A). In the control group, the infused
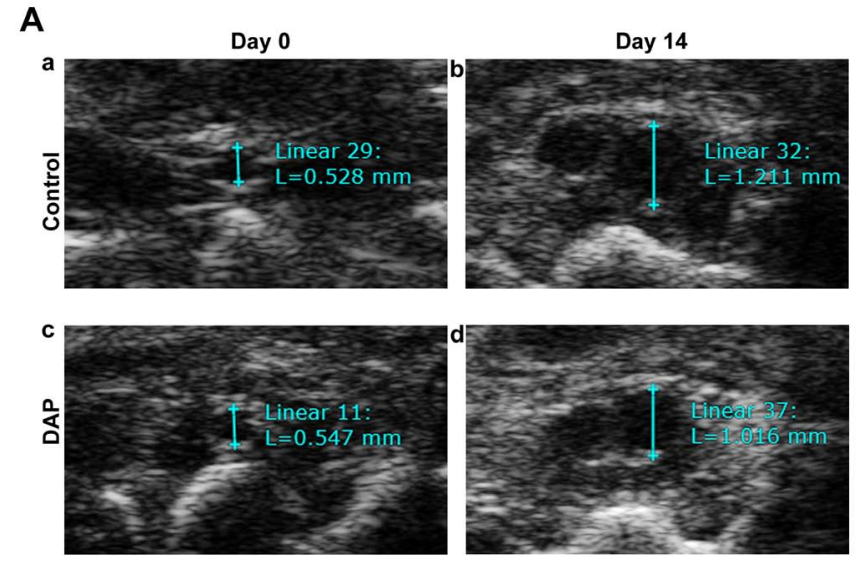

B

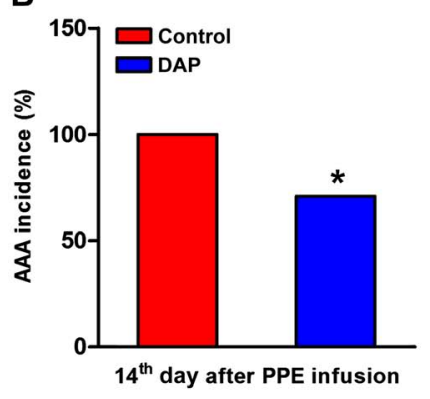

C

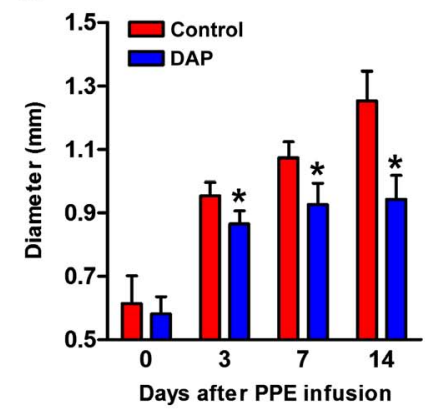

Figure 1. Effect of DAP treatment on experimental AAA formation and development. (A) Representative ultrasonographic images of aortas in the control and DAP treatment groups prior to and 14 days after intra-aortic infusion of PPE. (B) AAA incidence in each group on day 14 after PPE administration. (C) Maximum diameters of each group prior to and at days 3, 7 and 14 after PPE infusion. $n=6-7$ mice/group. ${ }^{*} \mathrm{P}<0.05$ vs. control group. AAA, abdominal aortic aneurysm; DAP, Daphnetin; PPE, porcine pancreatic.

abdominal aorta sustained enlargement in a time-dependent manner. In the DAP treatment group, aortic enlargement was significantly decreased compared with the control group. The maximum aortic diameter decreased significantly compared with that of the control group at each checkpoint after the operation (Fig. 1C). In the DAP treatment group, aneurysm incidence ( $>50 \%$ baseline diameter increase) was significantly decreased compared with the control on the 14th day after operation (71 vs. $100 \%$, DAP group vs. vehicle group; $\mathrm{P}<0.05$; Fig. 1B).

DAP treatment attenuates medial elastin and SMC destruction. To further investigate DAP-mediated protection against experimental AAA, changes in the aortic tissues at the morphological level were observed. Medial elastin and SMC destruction are the main histological characteristics of clinical and experimental AAAs $(10,40)$. In the DAP treatment group, SMCs stained with $\alpha$-SMA were degenerated and had almost fully disappeared in the aortic mural (Fig. 2A). The degeneration of SMCs was significantly decreased in the presence of DAP $(20 \mathrm{mg} / \mathrm{kg} / \mathrm{day})$. Furthermore, in the control group, elastin stained with EVG was degraded and had almost fully disappeared in the aortic mural (Fig. 2B). However, in the DAP treatment group, the EVG-stained elastic lamellae exhibited partial aortic elastin preservation, which was determined by semi-quantitative analysis $(\mathrm{P}<0.05)$. 
A

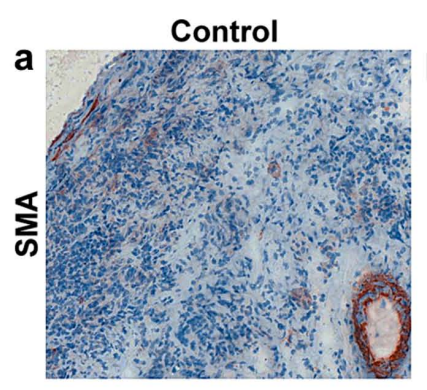

c

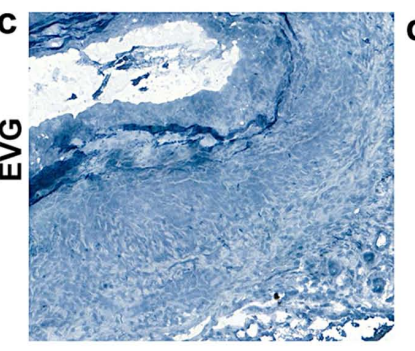

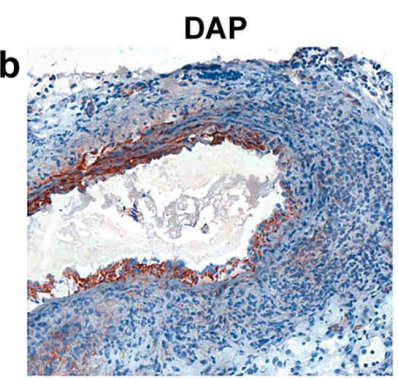

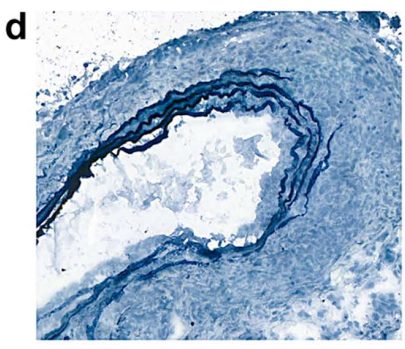

B a
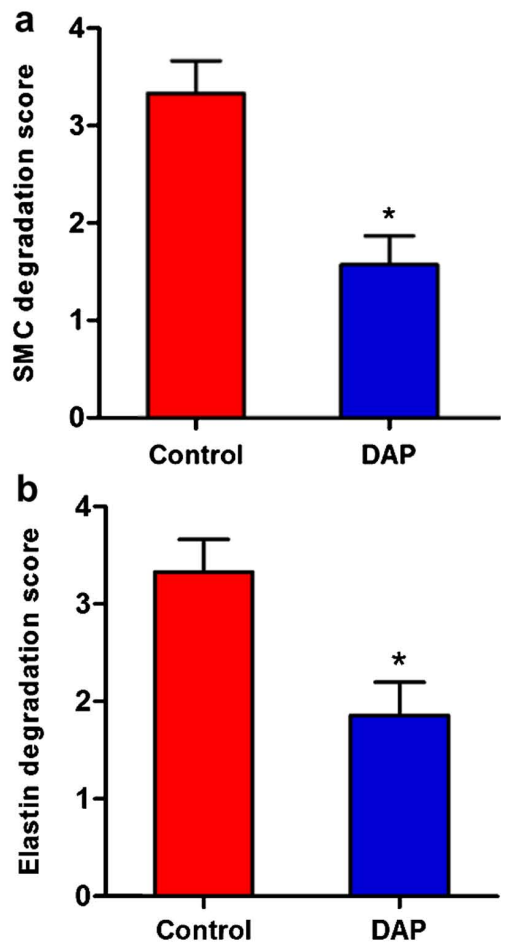

Figure 2. Effect of DAP treatment on medial SMCs and elastin destruction in the experimental AAA model. Mural elastin and smooth muscle cell degradation were graded from I (mild) to IV (severe). (A) Representative images of $\alpha$-SMA (a, b) and EVG (c, d) staining of SMCs and elastin, respectively, in the control and DAP treatment groups. (B) Mean scores of mural SMC degradation (a) and elastin degradation (b) in each group. $\mathrm{n}=6-7 \mathrm{mice} / \mathrm{group}$. ${ }^{*} \mathrm{P}<0.05 \mathrm{vs.} \mathrm{control}$ group. AAA, abdominal aortic aneurysm; DAP, Daphnetin; SMC, smooth muscle cell; EVG, Elastica van Gieson; $\alpha$-SMA, $\alpha$-smooth muscle actin.

Therefore, the preservation of medial elastin and SMC cellularity of aortic wall are the mural structural factors for explaining reduced aortic diameter enlargement in DAP-treated mice. These findings suggested that DAP may protect against AAA by attenuating aortic elastin and SMC destruction.

DAP treatment attenuates mural leukocyte infiltration. Mural leukocyte accumulation is another pathologic hallmark of AAA disease $(10,40)$. DAP is an anti-inflammatory agent used in inflammation-related diseases. To evaluate the influence of DAP treatment on mural inflammation, leukocyte infiltration was detected. CD68, CD8 and B220 staining was performed on aortic samples. As presented in Fig. 3, significant accumulation of macrophages and $\mathrm{T}$ and $\mathrm{B}$ lymphocytes was present in the media and adventitia in the control group. However, in the DAP treatment group, the increased numbers of mural macrophages, $T$ cells and $B$ cells were significantly decreased, which indicated that mural inflammation was attenuated. These results indicated that DAP treatment may limit experimental AAA progression in part by diminishing aortic accumulation of proinflammatory leukocytes.

DAP treatmentattenuates mural angiogenesis. Neoangiogenesis is an important histopathological feature of AAA (41-43). The influence of DAP treatment on mural angiogenesis was therefore evaluated, and CD31 staining was performed on aortic samples. As presented in Fig. 4, the quantity of mural CD31 ${ }^{+}$neovessels decreased significantly in the DAP treatment group compared with the control group. These results suggested that DAP treatment may suppress AAA by impairing mural angiogenesis during aneurysm formation and progression.

\section{Discussion}

DAP is a natural product extracted from Daphne odora var with anti-inflammatory, antioxidant and antitumour effects (21-25). Liu et al (24) reported that DAP protects against cerebral ischaemia/reperfusion injury in mice via inhibition of inflammatory responses. Yao et al (44) demonstrated that DAP has therapeutic effects on an autoimmune arthritis model by modulating inflammation. Yu et al (31) found that DAP possesses some anti-inflammatory activities in endotoxin-induced lung injury. Furthermore, Liu et al (26) reported that DAP has protective effects on severe acute pancreatitis in a rat model by inhibiting the expression of inflammatory cytokines. Ji et al (32) demonstrated that DAP ameliorates experimental colitis by modulating microbiota composition and the Treg/Th17 balance. Currently, an increasing number of studies have demonstrated that DAP serves a crucial role in the prevention and treatment of inflammation-related diseases due to its powerful anti-inflammatory activities (33-35).

The pathogenesis of AAA is characterized by inflammation with leukocyte infiltration, degradation of extracellular matrix and depletion of vascular smooth muscle cells $(10,40)$. Local chronic inflammation of the aortic wall has been implicated in the formation, development and rupture of AAA (1). Leukocytes are the principal effector cells of aneurysmal disease, contributing to aortic degradation via the production of extracellular MMPs, reactive oxygen species and proinflammatory cytokines. Leukocyte accumulation in the aortic wall is an early feature of PPE-induced AAAs and is present throughout the process of AAA. The present study demonstrated that high numbers of macrophages and $\mathrm{T}$ and 
A

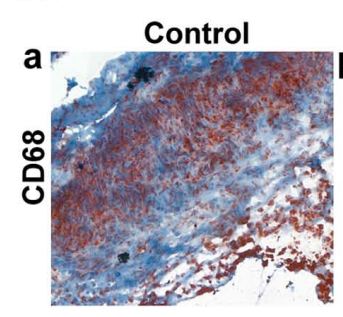

C

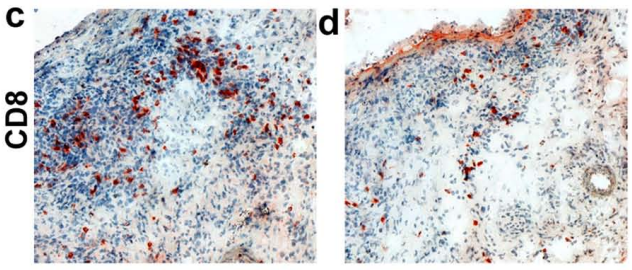

e

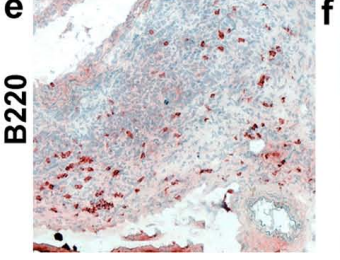

B
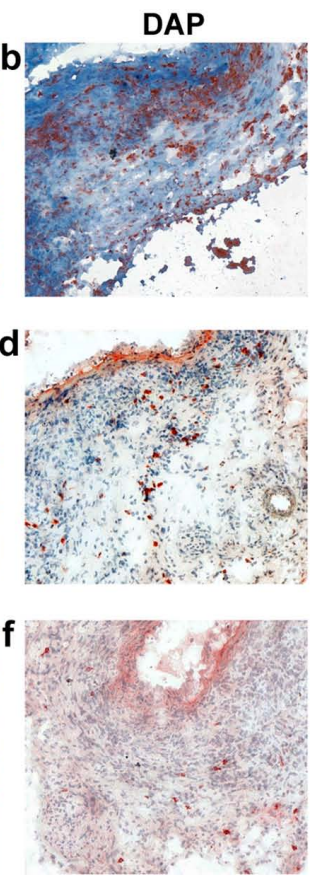
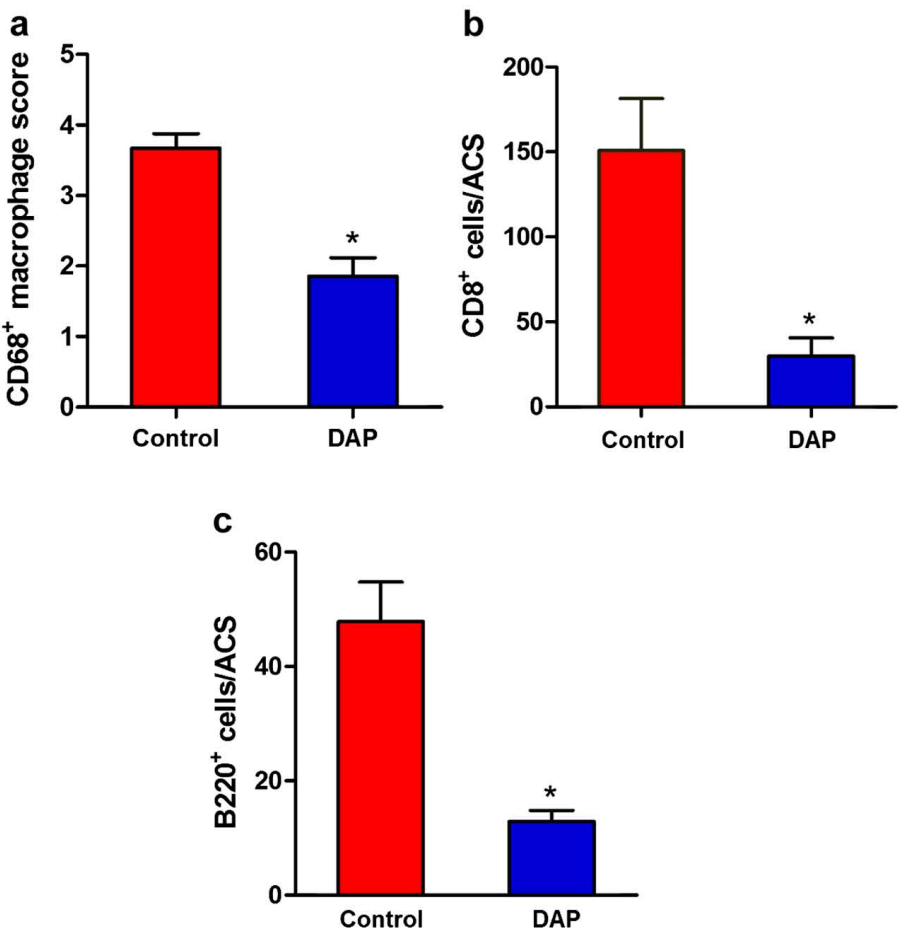

Figure 3. Effect of DAP treatment on mural leukocyte infiltration in the experimental AAA model. Mural macrophage accumulation was graded from I (mild) to IV (severe). (A) Representative immunostaining images of CD68+ macrophages (a, b), CD8+ T cells (c, d) and B220+ B cells (e, f) in the control and DAP treatment groups. (B) Mean scores of mural macrophages (a), T (b) and B cells (c) in each group were quantified as CD68+ cells, CD8+ cells and B220+ cells per ACS. $\mathrm{n}=6-7$ mice/group. ${ }^{*} \mathrm{P}<0.05$ vs. control group. ACS, aortic cross section; DAP, Daphnetin.

A

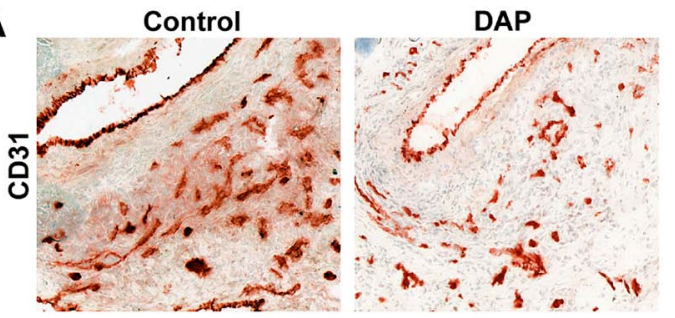

B

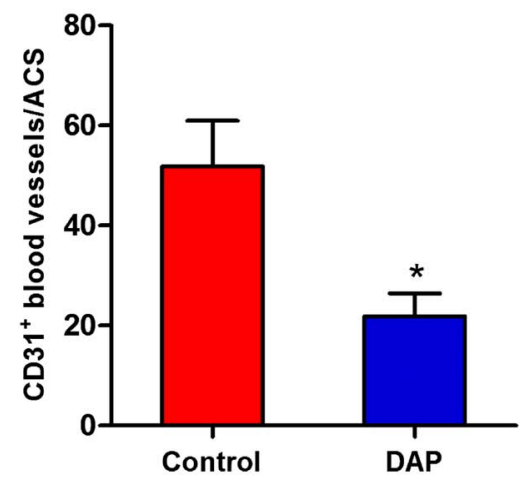

Figure 4. Effect of DAP treatment on aortic wall angiogenesis (A) Representative immunostaining images of CD31+ blood vessels in the control and DAP treatment groups. (B) Mean scores of mural angiogenesis in each group were quantified as $\mathrm{CD} 31^{+}$blood vessels per ACS. $n=6-7$ mice group. ${ }^{*} \mathrm{P}<0.05$ vs. control group. ACS, aortic cross section; DAP, Daphnetin.

B lymphocytes assembled in the media and adventitia in experimental AAAs. Mural macrophages and lymphocytes secrete various types of inflammatory cytokines and induce mural injury and subsequent aneurysm formation $(10,12,40)$. Previous studies have demonstrated that increased levels of inflammatory cytokines, including interleukin (IL)-1 $\beta$, IL- 6 and tumour necrosis factor (TNF)- $\alpha$, are positively correlated with AAA formation and expansion $(12,16,45,46)$. Lindberg et al (12) investigated the relationship between AAA and the inflammatory biomarkers IL-6, TNF- $\alpha$, endothelin-1 and soluble urokinase-type plasminogen activator receptor and reported that inflammatory cytokines play important roles in AAA progression. Xiong et al (13) reported that blocking TNF- $\alpha$ attenuates aneurysm formation in a murine model. De et al (14) found that systemic blockade of monocyte chemoattractant protein 1 inhibits aortic aneurysm formation in Ang II-induced AAA in apolipoprotein E-deficient mice. Mural inflammation serves therefore a crucial role in AAA formation and progression.

In the present study, following DAP treatment, the increased numbers of mural macrophages, $\mathrm{T}$ and $\mathrm{B}$ cells were significantly attenuated in the aortic wall. Previous studies also reported that DAP inhibits the infiltration of inflammatory cells to alleviate inflammatory injury in mice $(25,31,44)$. The results of DAP inhibiting the infiltration of inflammatory cells from the current study were consistent with previous studies. These results indicated that DAP may prevent the formation and progression of AAA via anti-inflammatory effects. Furthermore, treatment with DAP resulted in a significant reduction in mural neovessels. Neoangiogenesis is an important histopathological feature of AAA (41-43). Previous studies demonstrated that aortic mural neovascularization serves a key role in AAA progression and rupture (47-49). Kaneko et al (50) found that inhibition of vascular endothelial growth factor A(VEGF-A) by soluble VEGF-A receptor-1 attenuates experimental AAA 
development. Vijaynagar et al (48) reviewed studies on the role of angiogenesis in AAA and demonstrated that angiogenesis plays important roles in AAA progression. Recently, anti-angiogenesis therapy has been used as a potential intervention to treat AAA $(48,51,52)$. DAP treatment may therefore suppress AAA via impaired mural leukocyte infiltration and angiogenesis during aneurysm formation and progression.

The present study aimed to examine the influence of DAP on the formation and progression of experimental AAAs; however, this study had some limitations. A previous study reported that DAP inhibits the TLR4/NF- $\mathrm{KB}$ signalling pathway $(24,53)$. Liu et al $(24)$ found that DAP protects against cerebral ischaemia/reperfusion injury in mice via inhibition of the TLR4/NF- $\kappa B$ signalling pathway. Song et al (53) demonstrated that DAP downregulates the activation of concanavalin A-induced NF- $\mathrm{KB}$ signal transduction pathways in mouse $\mathrm{T}$ lymphocytes. A previous study has also found that TLR4/NF- $\mathrm{kB}$ signalling pathway is activated in AAA and mediates AAA formation and progression (54). DAP may therefore suppress experimental AAA by inhibiting the TLR4/NF- $\kappa B$-mediated inflammatory signalling pathway, and further investigation is thus required. In addition, the dose-range experiments and the clinical dose for humans was not determined in the current study. Therefore, substantial work will be required before clinical trials.

In conclusion, the findings from the present study suggested that DAP attenuated AAA formation and progression. This inhibitory effect may be mediated by inhibition of mural leukocyte infiltration and angiogenesis. These results suggested that DAP may be considered as a clinical candidate in early AAA disease suppression.

\section{Acknowledgements}

Not applicable.

\section{Funding}

No funding was received.

\section{Availability of data and materials}

The datasets used and/or analysed during the current study are available from the corresponding author on reasonable request.

\section{Authors' contributions}

$\mathrm{CH}$ and LW conceived and designed the experiments. SX, LM, HG, SG and LW performed the experiments. SX and CH analysed the data. $\mathrm{CH}$ wrote the manuscript. All authors read and approved the final manuscript.

\section{Ethics approval and consent to participate}

All experimental protocols were approved by the Medical Ethics Committee of Shandong Shanxian Central Hospital (Shanxian, China; approval no. IACUC A1027). All animals were cared for in accordance with the recommendations of the Guide for the Care and Use of Laboratory Animals of the National Institutes of Health (28).

\section{Patient consent for publication}

Not applicable.

\section{Competing interests}

The authors declare that they have no competing interests.

\section{References}

1. Golledge J, Muller J, Daugherty A and Norman P: Abdominal aortic aneurysm: Pathogenesis and implications for management. Arterioscler Thromb Vasc Biol 26: 2605-2613, 2006.

2. Miyake T and Morishita R: Pharmacological treatment of abdominal aortic aneurysm. Cardiovasc Res 83: 436-443, 2009.

3. Rouer M, Xu BH, Xuan HJ, Tanaka H, Fujimura N, Glover KJ, Furusho Y, Gerritsen M and Dalman RL: Rapamycin limits the growth of established experimental abdominal aortic aneurysms. Eur J Vasc Endovasc Surg 47: 493-500, 2014.

4. Greenhalgh RM, Brown LC, Kwong GP, Powell JT and Thompson SG: EVAR trial participants: Comparison of endovascular aneurysm repair with open repair in patients with abdominal aortic aneurysm (EVAR trial 1), 30-day operative mortality results: Randomised controlled trial. Lancet 364: 843-848, 2004.

5. Badger S, Forster R, Blair PH, Ellis P, Kee F and Harkin DW: Endovascular treatment for ruptured abdominal aortic aneurysm. Cochrane Database Syst Rev 5: CD005261, 2017.

6. Anderson RN: Deaths: Leading causes for 2000. Natl Vital Stat Rep 50: 1-85, 2002.

7. Xuan H, Xu B, Wang W, Tanaka H, Fujimura N, Miyata M, Michie SA and Dalman RL: Inhibition or deletion of angiotensin II type 1 receptor suppresses elastase-induced experimental abdominal aortic aneurysms. J Vasc Surg 67: 573-584.e2, 2018.

8. Lederle FA, Wilson SE, Johnson GR, Reinke DB, Littooy FN, Acher CW, Ballard DJ, Messina LM, Gordon IL, Chute EP, et al: Immediate repair compared with surveillance of small abdominal aortic aneurysms. N Engl J Med 346: 1437-1444, 2002.

9. Rehm JP, Grange JJ and Baxter BT: The formation of aneurysms. Semin Vasc Surg 11: 193-202, 1998.

10. Michel JB, Martin-Ventura JL, Egido J, Sakalihasan N, Treska V, Lindholt J, Allaire E, Thorsteinsdottir U, Cockerill G and Swedenborg J: FAD EU consortium: Novel aspects of the pathogenesis of aneurysms of the abdominal aorta in humans. Cardiovasc Res 90: 18-27, 2011.

11. Li G, Yang L, Yuan H, Liu Y,He Y, Wu X and Jin X: Cold-inducible RNA-binding protein plays a central role in the pathogenesis of abdominal aortic aneurysm in a murine experimental model. Surgery 159: 1654-1667, 2016.

12. Lindberg $\mathbf{S}$, Zarrouk $\mathbf{M}$, Holst $\mathbf{J}$ and Gottsater A: Inflammatory markers associated with abdominal aortic aneurysm. Eur Cytokine Netw 27: 75-80, 2016.

13. Xiong W, MacTaggart J, Knispel R, Worth J, Persidsky Y and Baxter BT: Blocking TNF-alpha attenuates aneurysm formation in a murine model. J Immunol 183: 2741-2746, 2009.

14. de Waard V, Bot I, de Jager SC, Talib S, Egashira K, de Vries MR, Quax PH, Biessen EA and van Berkel TJ: Systemic MCP1/CCR2 blockade and leukocyte specific MCP1/CCR2 inhibition affect aortic aneurysm formation differently. Atherosclerosis 211: 84-89, 2010.

15. Zhu H, Qu X, Zhang C and Yu Y: Interleukin-10 promotes proliferation of vascular smooth muscle cells by inhibiting inflammation in rabbit abdominal aortic aneurysm. Int J Clin Exp Pathol 12: 1260-1271, 2019.

16. Hao Q, Chen X, Wang X, Dong B and Yang C: Curcumin attenuates angiotensin ii-induced abdominal aortic aneurysm by inhibition of inflammatory response and ERK signaling pathways. Evid Based Complement Alternat Med 2014: 270930, 2014.

17. Sikora E, Scapagnini G and Barbagallo M: Curcumin, inflammation, ageing and age-related diseases. Immun Ageing 7: 1, 2010. 
18. Sunagawa Y, Wada H, Suzuki H,Sasaki H,Imaizumi A,Fukuda H, Hashimoto T, Katanasaka Y, Shimatsu A, Kimura T, et al: A novel drug delivery system of oral curcumin markedly improves efficacy of treatment for heart failure after myocardial infarction in rats. Biol Pharm Bull 35: 139-144, 2012.

19. Fan J, Li X, Yan YW, Tian XH, Hou WJ, Tong H and Bai SL: Curcumin attenuates rat thoracic aortic aneurysm formation by inhibition of the c-Jun N-terminal kinase pathway and apoptosis Nutrition 28: 1068-1074, 2012.

20. Kaluza J, Stackelberg O, Harris HR, Bjorck M and Wolk A Anti-inflammatory diet and risk of abdominal aortic aneurysm in two Swedish cohorts. Heart 105: 1876-1883, 2019.

21. Qi Z, Qi S, Gui L, Shen L and Feng Z: Daphnetin protects oxidative stress-induced neuronal apoptosis via regulation of MAPK signaling and HSP70 expression. Oncol Lett 12: 1959-1964, 2016

22. Fylaktakidou KC, Hadjipavlou-Litina DJ, Litinas KE and Nicolaides DN: Natural and synthetic coumarin derivatives with anti-inflammatory/antioxidant activities. Curr Pharm Des 10 3813-3833, 2004

23. Venugopala KN, Rashmi V and Odhav B: Review on natural coumarin lead compounds for their pharmacological activity. Biomed Res Int 2013: 963248, 2013.

24. Liu J, Chen Q, Jian Z, Xiong X, Shao L, Jin T, Zhu X and Wang L: Daphnetin protects against cerebral ischemia/reperfusion injury in mice via inhibition of TLR4/NF- $\kappa \mathrm{B}$ signaling pathway. Biomed Res Int 2016: 2816056, 2016.

25. Tu L, Li S, Fu Y, Yao R, Zhang Z, Yang S, Zeng X and Kuang N: The therapeutic effects of daphnetin in collagen-induced arthritis involve its regulation of Th17 cells. Int Immunopharmacol 13: 417-423, 2012.

26. Liu ZY, Liu J, Zhao KL, Wang LK, Shi Q, Zuo T, Liu TY, Zhao L and Wang WX: Protective effects of daphnetin on sodium taurocholateinduced severe acute pancreatitis in rats. Mol Med Rep 9: $1709-1714,2014$

27. Du G, Tu H, Li X, Pei A, Chen J, Miao Z, Li J, Wang C, Xie H, $\mathrm{Xu} \mathrm{X}$ and Zhao H: Daphnetin, a natural coumarin derivative, provides the neuroprotection against glutamate-induced toxicity in HT22 cells and ischemic brain injury. Neurochem Res 39 269-275, 2014

28. Zhi J, Duan B, Pei J, Wu S and Wei J: Daphnetin protects hippocampal neurons from oxygen-glucose deprivation-induced injury. J Cell Biochem 120: 4132-4139, 2019.

29. Chen X, Kuang N, Zeng X, Zhang Z, Li Y, Liu W and Fu Y: Effects of daphnetin combined with Bcl2siRNA on antiapoptotic genes in synovial fibroblasts of rats with collageninduced arthritis. Mol Med Rep 17: 884-890, 2018.

30. Shu K, Kuang N, Zhang Z, Hu Z, Zhang Y, Fu Y and Min W: Therapeutic effect of daphnetin on the autoimmune arthritis through demethylation of proapoptotic genes in synovial cells. J Transl Med 12: 287, 2014

31. Yu WW, Lu Z, Zhang H, Kang YH, Mao Y, Wang HH, Ge WH and Shi LY: Anti-inflammatory and protective properties of daphnetin in endotoxin-induced lung injury. J Agric Food Chem 62: 12315-12325, 2014

32. Ji J, Ge X, Chen Y, Zhu B, Wu Q, Zhang J, Shan J, Cheng H and Shi L: Daphnetin ameliorates experimental colitis by modulating microbiota composition and $\mathrm{T}_{\mathrm{reg}} / \mathrm{T}_{\mathrm{h}} 17$ balance. FASEB $\mathrm{J} 33$ : 9308-9322, 2019.

33. Li M, Shi X, Chen F and Hao F: Daphnetin inhibits inflammation in the NZB/W F1 systemic lupus erythematosus murine model via inhibition of NF- $\kappa \mathrm{B}$ activity. Exp Ther Med 13: 455-460, 2017.

34. Wang D, Lu Z, Zhang H, Jin SF, Yang H, Li YM and Shi LY: Daphnetin alleviates experimental autoimmune encephalomyelitis via regulating dendritic cell activity. CNS Neurosci Ther 22: 558-567, 2016.

35. Shen L, Zhou T, Wang J, Sang X, Lan L, Luo L and Yin Z: Daphnetin reduces endotoxin lethality in mice and decreases LPS-induced inflammation in Raw264.7 cells via suppressing JAK/STATs activation and ROS production. Inflamm Res 66: $579-589,2017$

36. Guide for the Care and Use of Laboratory Animals. In: National Research Council of the National Academes. 8th edition. Washington, DC, 2011.
37. Fujimura N, Xiong J, Kettler EB, Xuan H, Glover KJ, Mell MW, $\mathrm{Xu} B$ and Dalman RL: Metformin treatment status and abdominal aortic aneurysm disease progression. J Vasc Surg 64: 46-54. e8, 2016.

38. Iida Y, Xu B, Xuan H, Glover KJ, Tanaka H, Hu X, Fujimura N, Wang W, Schultz JR, Turner CR and Dalman RL: Peptide inhibitor of CXCL4-CCL5 heterodimer formation, MKEY, inhibits experimental aortic aneurysm initiation and progression. Arterioscler Thromb Vasc Biol 33: 718-726, 2013.

39. Iida Y, Xu B, Schultz GM, Chow V, White JJ, Sulaimon S, Hezi-Yamit A, Peterson SR and Dalman RL: Efficacy and mechanism of angiotensin II receptor blocker treatment in experimental abdominal aortic aneurysms. PLoS One 7: e49642, 2012.

40. Halloran BG and Baxter BT: Pathogenesis of aneurysms. Semin Vasc Surg 8: 85-92, 1995.

41. Wolanska M, Bankowska-Guszczyn E, Sobolewski K and Kowalewski R: Expression of VEGFs and its receptors in abdominal aortic aneurysm. Int Angiol 34: 520-528, 2015

42. Trollope AF and Golledge J: Angiopoietins, abdominal aortic aneurysm and atherosclerosis. Atherosclerosis 214: 237-243, 2011.

43. Li ZZ and Dai QY: Pathogenesis of abdominal aortic aneurysms: Role of nicotine and nicotinic acetylcholine receptors. Mediators Inflamm 2012: 103120, 2012.

44. Yao R, Fu Y, Li S, Tu L, Zeng X and Kuang N: Regulatory effect of daphnetin, a coumarin extracted from Daphne odora, on the balance of Treg and Th17 in collagen-induced arthritis. Eur J Pharmacol 670: 286-294, 2011.

45. Juvonen J, Surcel HM, Satta J, Teppo AM, Bloigu A, Syrjälä H, Airaksinen J, Leinonen M, Saikku P and Juvonen T: Elevated circulating levels of inflammatory cytokines in patients with abdominal aortic aneurysm. Arterioscler Thromb Vasc Biol 17: 2843-2847, 1997.

46. Flondell-Site D, Lindblad B and Gottsater A: High levels of endothelin (ET)-1 and aneurysm diameter independently predict growth of stable abdominal aortic aneurysms. Angiology 61: 324-328, 2010

47. Chapple KS, Parry DJ, McKenzie S, MacLennan KA, Jones P and Scott DJ: Cyclooxygenase-2 expression and its association with increased angiogenesis in human abdominal aortic aneurysms. Ann Vasc Surg 21: 61-66, 2007.

48. Vijaynagar B, Bown MJ, Sayers RD and Choke E: Potential role for anti-angiogenic therapy in abdominal aortic aneurysms. Eur J Clin Invest 43: 758-765, 2013.

49. Choke E, Cockerill GW, Dawson J, Wilson RW, Jones A, Loftus IM and Thompson MM: Increased angiogenesis at the site of abdominal aortic aneurysm rupture. Ann NY Acad Sci 1085: 315-319, 2006

50. Kaneko H, Anzai T, Takahashi T, Kohno T, Shimoda M, Sasaki A, Shimizu H, Nagai T, Maekawa Y, Yoshimura K, et al: Role of vascular endothelial growth factor-A in development of abdominal aortic aneurysm. Cardiovas Res 91: 358-367, 2011

51. Xu B, Iida Y, Glover KJ, Ge Y, Wang Y, Xuan H, Hu X, Tanaka H, Wang W, Fujimura N, et al: Inhibition of VEGF (vascular endothelial growth factor)-a or its receptor activity suppresses experimental aneurysm progression in the aortic elastase infusion model. Arterioscler Thromb Vasc Biol 39: 1652-1666, 2019.

52. Ma X, Yao H, Yang Y, Jin L, Wang Y, Wu L, Yang S and Cheng K: miR-195 suppresses abdominal aortic aneurysm through the TNF- $\alpha /$ NF- $\kappa$ B and VEGF/PI3K/Akt pathway. Int J Mol Med 41: 2350-2358, 2018

53. Song B, Wang Z, Liu Y, Xu S, Huang G, Xiong Y, Zhang S, Xu L, Deng $X$ and Guan $S$ : Immunosuppressive activity of daphnetin, one of coumarin derivatives, is mediated through suppression of NF- $\kappa \mathrm{B}$ and NFAT signaling pathways in mouse T cells. PLoS One 9: e96502, 2014.

54. Lai CH, Wang KC, Lee FT, Tsai HW, Ma CY, Cheng TL, Chang BI, Yang YJ, Shi GY and Wu HL: Toll-like receptor 4 is essential in the development of abdominal aortic aneurysm. PLoS One 11: e0146565, 2016.

This work is licensed under a Creative Commons Attribution-NonCommercial-NoDerivatives 4.0 International (CC BY-NC-ND 4.0) License. 